\title{
Rūta KEMERE
}

Liepājas 5.vidusskola

\section{Alïda SAMUSEVIČA}

Liepaja Universität

\section{FREMDSPRACHENERWERB IM KONTEXT DER FÖRDERUNG DES MultilingualismUS IN LETTLAND}

Zusammenfassung. Die Publikation behandelt die Erkennung von Möglichkeiten zur Förderung des Fremdsprachenerwerbprozesses der Schüler in der Tätigkeit der Schulen Lettlands mit erweitertem Sprachunterricht. Die Tätigkeit der Schulen mit erweitertem Sprachunterricht ist eine bedeutende Ressource zur Förderung und Entwicklung des Multilingualismus, der in Globalisierungsverhältnissen, wenn die geographische Mobilität wächst, die Qualität der sozialen Wechselwirkung der Gesellschaft entwickelt, indem der Übergang von einer homogenen Kultur und eines homogenen Wertsystems auf Pluralismus der Kultur und auf Heterogenität gefördert wird. Die Tätigkeit der Schulen mit erweitertem Sprachunterricht strukturiert das pädagogische System, das elastisch funktioniert und offen für Bildungsreformen auch beim Wandel des Paradigmas im Sprachenerwerb ist.

Ziel der Publikation - Einschätzung von Optimierungsmöglichkeiten der pädagogischen Tätigkeit an Sprachdiplomschulen (DSD Schulen) zur Aktualisierung der Qualität des Fremdsprachenerwerbs im Kontext der Förderung des Multilingvismus. Die Autorinnen analysieren in der Publikation die Richtlinien der Fremdsprachenbildung in Lettland in der Stabilisierung der Positionen des Multilingualismus, beurteilen die organisatorischen und pädagogischen Aspekte in der Tätigkeit der DSD Schulen zur Förderung des Multilingualismus - mit Schwerpunkt auf die Aktualität der didaktischen und pädagogischen Vielfalt. Bei der Bestimmung der innovativen pädagogischen Ansätze ist die Beschreibung und die Analyse von Optimierung der pädagogischen Tätigkeit der DSD Schulen zur Aktualisierung der deutschen Sprache sehr wichtig.

In der Publikation werden die Ergebnisse der empirischen Forschung über Motivation und Qualität des Fremdsprachenerwerbs analysiert, Verhältnis und Meinungen der Jugendlichen aus dem Standpunkt der Entwicklung einer multikulturellen Gesellschaft eingeschätzt. Es werden grundlegende Voraussetzungen für die Förderung des Multilingualismus genannt, die die Positionen des Multilingualismus durch Unterstützung von Schulen mit erweitertem Sprachunterricht in Lettland und durch Akzeptanz der demokratischen und didaktischen Initiativen von diesen Schulen als Zentren der Vielsprachigkeit stärken können. Es ist wichtig dabei, einen gleichzeitigen Erwerb von mehreren Fremdsprachen den Schülern anzubieten, die erste Fremdsprache frei wählen zu lassen, mit dem Fremdsprachenunterricht schon in der ersten Klasse zu beginnen und das Bewertungssystem im Sprachunterricht zu 
vervollkommnen, sodass die Einschätzung der Sprachkompetenzen der Schüler zum Förderungsmittel der Schülermotivation im Sprachenerwerb werden kann.

Die Ergebnisse der empirischen Forschung zeugen anschaulich davon, dass die Motivation der Schüler an den Schulen mit erweitertem Sprachunterricht, ihre Offenheit mehreren Fremdsprachen gegenüber, deren zielstrebiger und qualitativer Erwerb schon in der Schulzeit und kreativer Gebrauch im weiteren Leben die wichtigsten Voraussetzungen im Sprachenerwerb präsentieren, die aktuell für Jugendliche sind und in großem Maße die Qualität des Multilingualismus in der Gesellschaft Lettlands bestimmen werden. Die Rolle des Fremdsprachenunterrichts in Lettland hoch genug einzuschätzen- das ist eine wichtige vom Paradigma der demokratischen und humanen Bildung bedingte Aufgabe, die sich an der Unikalität jeder Persönlichkeit, an Ganzheit und Einzigartigkeit der Sprache orientiert.

Schlüsselwörter: Schüler, Fremdsprachenerwerb, Schulen mit erweitertem Sprachunterricht, Förderung des Multilingualismus.

\section{Einleitung}

Die immer aktivere Mobilität in der Europäischen Union, der Prozess der Globalisierung, ein einheitlicher Markt sind die Hauptgründe, warum die Einwohner mit einer immer größeren Sprachenvielfalt in Berührung kommen. Die EU hat ein konkretes Ziel zur Stärkung der linguistischen Vielfalt gestellt - d.h. das Prinzip von Barcelona: jeder Einwohner der EU sollte seine Muttersprache und wenigstens noch zwei Sprachen beherrschen (Valodas situācija Latvijā: 2004-2010, 2010, s. 13).

In den meisten Staaten der Europäischen Union ist der Erwerb von zwei Fremdsprachen wenigstens ein Jahr lang in der Pflichtschulbildung obligat für alle Schüler. Im Schuljahr 2009/2010 haben durchschnittlich 60,8\% Schüler in der ersten Stufe der mittleren Bildung zwei oder mehr Sprachen gelernt; das ist um $14,1 \%$ mehr als im Jahr 2004/2005. In der zweiten Stufe der mittleren Bildung besteht zwischen Ländern ein großer Unterschied in der Zahl von Schülern, die zwei oder mehr Fremdsprachen in der allgemeinen $(59,4 \%)$ und in der beruflichen (39,4\%) Ausbildung lernen (Pamatrādītāji par valodu mācīšanu Eiropas skolās, 2012, s. 2).

Der Multilingualismus in Lettland hat im Vergleich zu anderen EU- Ländern ein hohes Niveau erreicht. In Lettland beherrschen 95\% der Einwohner eine FS, $51 \%$ zwei FS und 14\% drei FS: dank der Personenzahl, die eine oder mehrere Fremdsprachen beherrschen, ist Lettland an dritter Stelle unter anderen EU- Ländern. (Sabiedrības integrācijas politikas pamatnostādnes 2010-2019 gadam, 2010). Das kann dadurch erklärt werden, dass die Letten ziemlich gute Russischkenntnisse, die sie in der Zeit der UdSSR erworben haben, besitzen. Die soziokulturelle Heterogenität ist eine Herausforderung für eine beliebige Gesellschaft, die sowohl politisch als auch wirtschaftlich eine lange Zeit in Isoliertheit gelebt hat und die sich zielbewusst an Entwicklungsdimensionen der globalen Welt beteiligt.

Zur Zeit werden in europäischen Ländern Studien über die Motive des Sprachenerwerbs unter Einwohnern durchgeführt, denn die Motivation ist der wichtigste 
Stimulus beim Sprachlernen. Es werden Ergebnisse zusammengefasst, um feststellen zu können, ob die Haupttendenz der Gesellschaft ist, sich auf den Erwerb nur einer Fremdsprache zu beschränken (also Monolingualismus) oder den Multilingualismus als einen positiven Wert zu wählen.

Die Agentur der Lettischen Sprache hat 2009 Personen, deren Muttersprache Lettisch ist, befragt. Die Antworten auf die Frage, welche Sprachen die Kinder an der Schule lernen sollten, sind traditionell- Englisch, Russisch, Deutsch und Französisch. Auf die Notwendigkeit der englischen Sprache an der Schule weist die Mehrheit der Befragten hin (92\%), für Russisch entscheiden sich $70 \%$ der Befragten. Die Menschen mit höherem Bildungsniveau nennen häufiger Englisch, Deutsch und Französisch, seltener - Russisch. Der Englischunterricht ist öfter für jüngere als für ältere von Bedeutung (Valodas situācija Latvijā: 2004-2010, 2012, s. 21). Die Ergebnisse der Studie über die Situation des Fremdsprachenerwerbs in Schulen der baltischen Länder bestätigen, dass es in Estland und Litauen prozentuell mehr Schüler als in Lettland gibt, die Deutsch und Französisch lernen; in Lettland gibt es ein wenig mehr Schüler, die Englisch lernen (Key Data on Teaching Languages at School in Europe, 2008, s. 74). In Lettland haben die zentralisierte Deutschprüfung 2012, zum Beispiel, nur 309 Schüler gewählt. Die Angaben über die vorigen Jahren: 2011 - 453, 2010 - 506, 2009 - 734, 2008 - 801 (Mitrofanova, 2012, s. 2).

Die angeführte Statistik und die pädagogische Erfahrung der Autorinnen bestätigen ein aktuelles Problem in der Schulpraxis - eine krasse Senkung des Prestiges der deutschen Sprache unter Schülern. Dieses Problem kann in der Tat eine optimale Tätigkeit der DSD Schulen gefährden. Als Folge kann das reiche Spektrum der Vielsprachigkeit unter Studenten, unter jungen Spezialisten, in der ganzen Gesellschaft begrenzt werden.

Ziel der Publikation: Einschätzung von Optimierungsmöglichkeiten der pädagogischen Tätigkeit an Sprachdiplomschulen (DSD Schulen) zur Aktualisierung der Qualität des Fremdsprachenerwerbs im Kontext der Förderung von Multilingualismus.

Forschungsgegenstand der Publikation: pädagogisch linguistische und organisatorische Bedingungen zur Stabilisierung der weiteren Tätigkeit der DSD Schulen in Lettland im Kontext der Förderung von Multilingualismus.

\section{Sprachenbildungspolitik in Lettland zur Stabilisierung der Positionen des Multilingualismus}

Der Weg Lettlands zur Demokratisierung, der Wunsch sich im politischen und Kulturraum von Europa zu integrieren, hat in der Mitte der 90-er Jahre die Aufnahme von Elementen der Diversität in Schulprogramme gefördert: diverse Elemente der bürgerlichen Ausbildung sowie kulturelle und ethnische Elemente wurden als positiv eingeschätzt. Dazu haben auch die Richtlinien des Europarates und 
die Bildungspolitik der EU beigetragen (Valtenbergs, 2006, s. 281-312). In der Bildungspolitik Lettlands wurde trotzdem der Aspekt der Vielsprachigkeit nicht genug unterstützt, denn auf dem Niveau der Staatspolitik hat man das utilitäre Ziel der Sprache hervorgehoben. Das ist aber nur die dominierende Sichtweise der Globalisierung, in der man bloß eine Sprache - die Verkehrssprache Englischmonopolisieren möchte. Mit Rücksicht darauf, dass die Sprache zentrales Symbol der Identität eines jeden Ethnos ist, kann nur eine Sprache als internationales Kommunikationsmittel keinesfalls eine gegenseitige Verständigung sichern- nur eine vereinfachte und primitive Kommunikation. Multikulturelle Bildung setzt sich gegen verschiedenartige Diskriminierungsformen in der Gesellschaft ein, akzeptiert und bestätigt die Ideen des Pluralismus, darunter den ethnischen Pluralismus, Rassenpluralismus, Sprachenpluralismus, religiösen Pluralismus, Rassenpluralismus, Sprachenpluralismus, Religionspluralismus, Wirtschaftspluralismus und Geschlechtspluralismus (Laicāne, 2004, s. 2). Wenn die Idee des Multilingualismus vom Staat unterstützt wird, ist es leichter, den Multilingualismus auch im Bildungssystem zu realisieren. Eine aktuelle Tendenz im Sprachenerwerb an lettischen Schulen war aber ein überhasteter Übergang auf die Einschließung der populärsten Fremdsprache (Englisch) ins Curriculum und Bemühungen, die Schulen gleichzumachen.

Die Sprachschulen werden oft für elitär gehalten: im Kontext des Multilingualismus gilt diese Annahme als positiv, denn das Angebot der Schulen mehr Fremdsprachen als in anderen Bildungsstätten zu lernen und sie qualitativ zu unterrichten entspricht den Prinzipien der Demokratie. Das Angebot von mehreren Sprachen bedeutet die Freiheit der Wahl. Die Prioritäten der DSD Schulen sind ein elitärer Effekt, der die Beachtung der Gesellschaft finden und einen qualitativen Sprachenerwerb sichern kann - unter Bedingung, dass an Multilingualismus auch die Gesellschaft interessiert ist und dass der Unterrichtsprozess aufgrund der Innovationen in der Bildung und in anderen Lebensbereichen der Gesellschaft optimiert wird. Das multikulturelle Umfeld, das chronologisch nach der vorher herrschenden, grundsätzlich homogenen Gesellschaftskultur folgt, lässt die Formulierung der Ziele im Bildungsgesetz auswerten, um feststellen zu können, ob sie der heutigen Situation und den Tendenzen in der Gesellschaft entspricht. Im Bildungswesen soll man sowohl mit raschen Änderungen in der Gesellschaft als auch mit Bedarf an Nachhaltigkeit rechnen (Mūrnieks, 2011, s. 5). Da die Fremdsprachenkompetenzen immer aktueller werden, ist es wichtig, in jedem Land neben den gesamten europäischen Richtlinien die lokalen Ziele und Mittel in der Fremdsprachenbildung, die die Qualität im Erwerb von mehreren Sprachen sichern, bewusst zu werden.

Die Mehrheit der Eltern in Lettland verlangen, dass ihre Kinder an der Schule Englisch als erste Fremdsprache lernen, denn Englisch ist leichter zu erlernen und als globales Kommunikationsmittel ist das eine günstige Voraussetzung in der weiteren Bildungsstufe und im Beruf. Als erste Fremdsprache kann Englisch leider 
nicht motivieren, weitere Sprachen zu lernen. Auch in der „Europäischen Charta für Mehrsprachigkeit" wird betont: „Die zuerst erlernte Sprache sollte unter gegenwärtigen Umständen möglichst nicht Englisch sein" (Europäische Charta für Mehrsprachigkeit, 2005, s. 7). Sowohl auf Ebene des Informationsaustausches als auch auf emotionaler Ebene wird die Verständigung immer besser sein, wenn die Kommunikationspartner jeder die Sprache des anderen spricht, statt eine dritte Sprache verwenden - obwohl das eine globale Sprache ist. Der Multilingualismus bedeutet mehrere Sprachen zu beherrschen und nicht eine, mit der man in jedem Land und unter allen Umständen „manipulieren” kann. Deswegen für qualitativen Erwerb von mehreren Sprachen ist eine optimal ausgearbeitete Konzeption der regionalen Mehrsprachigkeit wichtig, die im Rahmen einer Ausbildungsform den Unterricht von zwei, drei und noch mehr Fremdsprachen anbietet, was der Fall der Schulen mit erweitertem Sprachunterricht ist. Der Erwerb von mehreren Fremdsprachen ist ein Unterstützungsfaktor für Positionen des Individuums und für dessen Integration in der multikulturellen, vielsprachigen Umwelt. Auf Basis von komplizierten Fremdsprachen (wie Deutsch, Französisch u.a.) ist es einfacher Englisch zu erlernen. Ungeachtet dessen, dass die erste FS kein Englisch ist, sind die Ergebnisse dieser Schulen in der zentralisierten Englischprüfung Lettlands sehr gut. Der deutsche Linguist F. G. König betont in seiner Forschung „Fremdsprachen lehren und lernen", dass zwischen Kenntnissen und Fähigkeiten in der Fremdsprache die Korrelation besteht und auf Basis des Vergleichs von Sprachstrukturen und von einzelnen Elementen nicht schwer ist, jede nächste Fremdsprache zu erlernen (Königs, 2000, s. 43).

\section{Optimierung der pädagogischen Tätigkeit an DSD Schulen zur Aktuali- sierung der deutschen Sprache}

Die DSD Schule ist eine optimale Forschungsbasis zur Erkennung der Motivation mehrere Sprachen zu erlernen, zur Sammlung von innovativen Verbesserungen, die einen qualitativen und vielfältigen Spracherwerb und nachhaltiges Wissen unter Jugendlichen fördern. Bei der Klassifizierung der Bildungsziele lassen sich die objektiv qualitativen, mit Sprachkompetenz verbundenen Ziele und die subjektiv qualitativen, mit Persönlichkeitsqualitäten verbundenen Ziele erkennen (Verhältnis, Wertorientierung, Überzeugung u.a.) Sowohl die objektiven als auch die subjektiven Ziele kann man anhand von Programmen und Materialien für die DSD Schulen verwirklichen. Progressive Methoden und innovative Lernstoffe für Schüler und Lehrer der DSD Schüler und Lehrer sind vorwiegend elektronisch erstellt. Computer und Internet sind zum wichtigen Unterrichtsmittel im Fremdsprachenerwerb der Europaländer geworden. Beim Wandel des Paradigmas im Fremdsprachenunterricht werden allmählich Prinzipien der Mehrsprachigkeitsdidaktik realisiert: die Sprachen werden nicht isoliert unterrichtet, sondern in gegenseitiger Wechselwirkung und Abhängigkeit und aufgrund von Lerninhalten aus anderen Schulfächern, woran Schüler Interesse zeigen. Das 
verlangt eine noch aktivere Kooperation zwischen Lehrern, eine gemeinsame Stoffverteilung u.a. Außerdem soll das behandelte Thema der Diskussionen in den Sprachstunden für die Schüler interessant, nützlich und adäquat sein (Didaktik der Mehrsprachigkeit, 2010). Das Potential jeder Klasse oder Lerngruppe ist unterschiedlich - das ist eine Mosaik, die man sorgfältig erforschen und professionell zu einer Einheit im Spracherwerb entwickeln soll, mittels deren die Schüler einander in Fragen helfen können, die sie besser beherrschen: das ist nicht bloß die Zusammenarbeit mit dem Lehrer, der die Lerntätigkeit an der Schule organisiert (Franceschini, 2009). Vertreter der Didaktik F. J. Meißner betont in seinen Publikationen die Notwendigkeit des fächerübergreifenden Prinzips: die Verbindung soll nicht nur zwischen Sprachen bestehen, sondern auch zwischen den exakten und humanitären Fächern. Diese Knotenpunkte zwischen den Sprachen und anderen Schulfächern hat er schon Etikette genannt. Von der ersten Etappe der Mehrsprachigkeitsdidaktik zeugen schon neue alternative Termini, wie „integrierter Fremdsprachenerwerb", "kooperative Didaktik" u.a. Auch im mehrsprachigen Brüssel wird weit der Begriff der Mehrsprachigkeitsdidaktik "didactique du plurilinguisme" verwendet (Meißner, 2004, s. 56). Wahrscheinlich verbindet sich mit der Mehrsprachigkeits- und Mehrkulturalitätsdidaktik und Neuen Technologien eine neue Lehr- und Lernkultur, welche die monolingualen Sichtweisen und Lehrpraxen in Teilen relativieren und ablösen (Meißner, 2004, p. 57). Die wichtigste Voraussetzung für einen optimalen Spracherwerb an der Schule ist die Motivation der Jugendlichen Sprachen zu erlernen, sowohl an der Schule als auch im außerschulischen Umfeld.

\section{Die Motivation der Schüler beim Fremdsprachenerwerb}

Die Schüler der Forschungsbasis (12 Klassen) äußern 2011 überzeugend ihre Meinung, dass die Schule wenigstens drei Fremdsprachen anbieten sollte. Die Änderungen in der politischen und wirtschaftlichen Situation in Lettland führen zur Notwendigkeit Fremdsprachen zu erlernen. Vor zwölf Jahren hat das in ihrer Forschungsarbeit "Linguistisches Verhältnis der Einwohner Lettlands" die Philologin D.Baltaiskalna festgestellt, sie betont auch, dass in "Lettland unter Vertretern der jüngeren Generation Trilinguismus zur Relaität geworden ist "(Baltaiskalna, 2001). Die Antworten von den Schülern über die Reihenfolge beim Erlernen der Sprachen sind unterschiedlich (siehe Tabelle 2). Wenn die instrumentale Motivation hauptsächlich pragmatischen Charakter hat und der Schüler an seine eigenen Vorteile auf dem Arbeitsmarkt und an seine Karriere interessiert ist, richtet sich die integrative Motivation auch auf die Anerkennung von Traditionen einer unbekannten Kultur, auf Kontakte mit Vertretern eines anderen Landes als mit Trägern dieser Kultur. 
Könnte man den Sprachunterricht an der Schule mit dem Erwerb eines weniger populären Sprache beginnen (Deutsch, Französisch) ? $(\mathrm{N}=101)$

\begin{tabular}{|c|c|c|c|c|}
\hline Unbedingt & Ja & Vielleicht & Nein & Weiß nicht \\
\hline $29 \%$ & $33 \%$ & $19 \%$ & $18 \%$ & $1 \%$ \\
\hline $26 \%$ & $49 \%$ & $20 \%$ & $4 \%$ & $1 \%$ \\
\hline
\end{tabular}

Die Ergebnisse der empirischen Forschung beweisen, dass das Verhältnis der heutigen Schüler zu den Sprachen Offenheit und Interessiertheit charakterisieren. Aus der Tabelle 3 folgt, dass Englisch als die populärste Sprache für $76 \%$ der befragten Schüler an der Forschungsbasis gilt. Deutsch ist für 56\% die zweitpopulärste Sprache und Russisch ist für 57\% die drittpopulärste Sprache. Englisch wurde 93 mal unter populärsten Sprachen genannt, Deutsch 75 mal, Russisch 56 mal. Die Statistik zeigt, dass auch andere Sprachen, die fakultativ gelernt werden, halten die Schüler für wichtig: 18 mal ist Französisch genannt (als die populärste Sprache in $11 \%$ der Fälle) und Spanisch 14 mal (als die populärste Sprache in $14 \%$ der Fälle).

Tabelle 2

Die populärsten Fremdsprachen unter Schülern der 12.Klassen an der. Forschungsbasis (an einer der DSD Schulen Lettlands $2010 / 2011)(N=101)$

\begin{tabular}{|c|c|c|c|c|}
\hline Englisch & $93 x$ & $1-71-76 \%$ & $2-15-16 \%$ & $3-7-8 \%$ \\
\hline Deutsch & $75 x$ & $1-10-13 \%$ & $2-42-56 \%$ & $3-23-31 \%$ \\
\hline Russisch & $56 x$ & $1-5-9 \%$ & $2-19-34 \%$ & $3-32-57 \%$ \\
\hline Französisch & $18 x$ & $1-2-11 \%$ & $2-7-39 \%$ & $3-9-50 \%$ \\
\hline Spanisch & $14 x$ & $1-2-14 \%$ & $2-7-50 \%$ & $3-5-36 \%$ \\
\hline
\end{tabular}

Die Befragung der Schüler im Jahr 2012 ( $N=78$ ) über die erworbenen Kenntnisse in den Fremdsprachen lässt dagegen schlussfolgern, dass es notwenig ist, den Sprachunterricht an der Schule zu vervollkommnen. Englisch beherrschen gut genug 44,9\%. Nur ein Befragter erkennt, dass er Englisch nicht genug beherrscht. Deutsch beherrschen weniger Schüler (17,9\%), der größte Teil der Befragten will die Deutschkenntnisse verbessern (65.4\%) und 10,3\% erkennen, dass sie schwache Deutschkenntnisse besitzen. Nur 6,4\% der Befragten können diese Sprache frei gebrauchen. 98,7\% der Befragten stellen fest, dass sie im außerschulischen Umfeld Englisch gut verstehen Nur 1,3\% antworten, dass sie Englisch nicht verstehen (Hör- und Leseverstehen). Deutsch verstehen weniger Schüler $(43,6 \%)$, in dieser Sprache fühlen sich unsicher 56,4\%. Die meisten der befragten Schüler lesen Internetartikel im Englischen und dabei verstehen die nötige Information $(96,2 \%)$. Cirka 4\% lesen keine Informationen im Internet in dieser Sprache. In der deutschen Sprache werden Internetseiten seltener gelesen: nur 32,1\% benutzen diese Gelegenheit. Fast $70 \%$ tun das nicht. Die Schüler werden darüber gefragt, ob sie in Zukunft noch stärkere Positionen des Monolonguismus prognosieren. 
Sie sind der Meinung, dass entscheidend für das Leben in einer multilingualen Gesellschaft die Beherrschung von mehreren Sprachen sein wird - d.h. Multilingualismus, das merken $84,6 \%$ der Befragten. 15,4\% prognosieren eine weitere Hegemonie des Englischen in allen Lebensbereichen. Es wurde auch die Meinung darüber geklärt, was richtiger ist: 78,2\% (61 Schüler) ziehen das Prinzip des Multilingualismus vor. Ein Fünftel der Befragten ist nicht dagegen, dass die Welt multilingual (englisch) spricht und hält das sogar für günstig. Das sind 20,5\%. In Interviews mit Schülern wurden die wichtigsten persön.

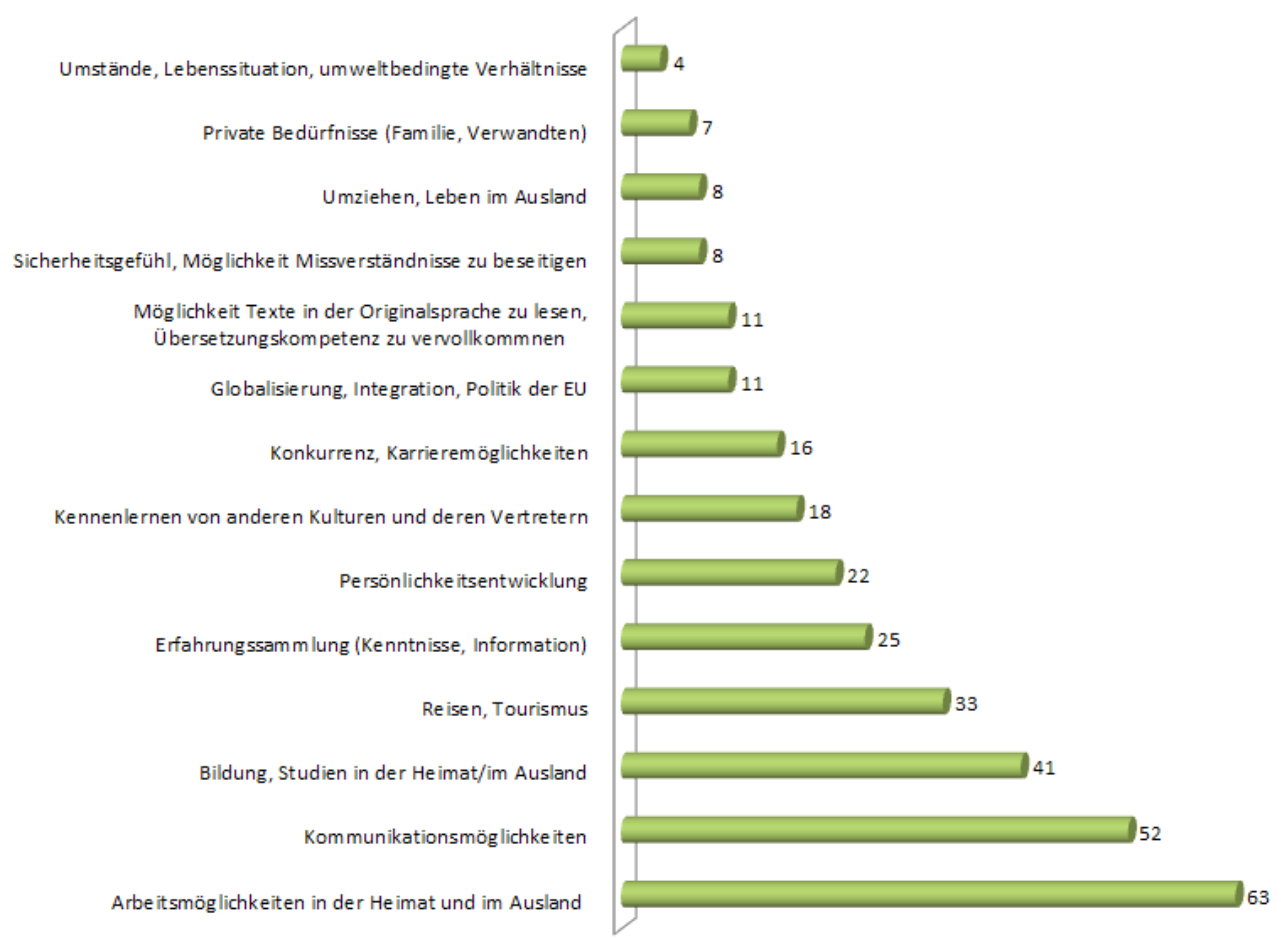

Figure 1. Motive des Sprachlernens der Schüler der 12.Klassen an der Forschungsbasis

Laut geäußerten Meinungen von Schülern sind die Fremdsprachenkenntnisse immer stärker mit den künftigen Chancen im Arbeitsmarkt, mit Perspektiven in der beruflichen Karriere, verbunden- auch mit der Anknüpfung von privaten Kontakten. Bei der Zusammenfassung der erwähnten Motive bildet sich ein bestimmtes systemisches Gesamtbild, das davon zeugt, dass die Schüler logisch und zielbewusst ihre persönlichen individuellen Bedürfnisse im Einklang mit Verhältnissen des äußeren Umfelds, die die Tendenzen der modernen Gesellschaft der Welt beeinflussen, bringen wollen. Das sind solche Verhältnisse wie Globalisierung und Notwendigkeit, sich in einer produktiven sozialen Kommunikation zu integrieren. 


\section{Schlussfolgerungen}

Die Schulen mit erweitertem Sprachunterricht (DSD Schulen) sind immer ein bedeutender Bestandteil des lettischen Bildungssystems gewesen. In der jetzigen Situation sind sie eine wichtige Ressource, die eine radikale Hilfe bei der Verbesserung von Fremdsprachenkenntnissen unter Jugendlichen ist. Die Aktualität einer multilingualen Gesellschaft fordert eine ununterbrochene Aktualisierung von Fremdsprachenkenntnissen und eine qualitative Alltagstätigkeit in Bildungsstätten und Institutionen, indem die Bedeutung der Sprachen in der Karriere und Kommunikation eines jeden konkreten Individuums neu ausgewertet wird. Für die Stärkung der Positionen des Multilingualismus in Lettland ist es wichtig, die Optimierung der Schulen mit erweitertem Sprachunterricht (DSD Schulen) sowie die demokratischen und didaktischen Initiativen von diesen Schulen als Zentren des Multilingualismus zu unterstützen.

Wesentlich beim Spracherwerb ist die Reihenfolge der Fremdsprachen. Deutsch aus der Sicht der Linguisten, der Vertreter von anderen Völkern, ist eine komplizierte Fremdsprache. Wenn der Schüler in den jüngeren Klassen die Sprachen lernt (und bereits ab 1.Klasse) und die Grundlagen einer Sprache spontan entdeckt, ohne speziell die Vokabel und Grammatik zu lernen, ist für ihn in den älteren Klassen viel einfacher andere, die relativ leichteren Sprachen, zu erlernen.

Ergebnisse der empirischen Forschung präsentieren anschaulich, dass die Motiviertheit der Schüler in den Schulen mit erweitertem Sprachunterricht, ihre Offenheit gegenüber mehreren Fremdsprachen, ihr zielstrebiger und qualitativer Erwerb von Sprachen in der Schulzeit und kreative Anwendung im weiteren Leben die bedeutendsten Voraussetzungen für den Sprachenerwerb sind. Diese Voraussetzungen sind aktuell für Jugendliche und in großem Maße stärken die Qualität des Multilingualismus in der Gesellschaft Lettlands.

\section{Quellen}

Baltaiskalna, D. (2001). Latvijas iedzīvotāju lingvistiskā attieksme. Rīga: LU, Latviešu valodas institūts. Retrieved from http://www.vvk.Iv/index.php?sadala $=131 \& i d=174$.

Didaktik der Mehrsprachigkeit. Retrieved from http://www.passepartout-sprachen. $\mathrm{ch} / . . . /$ didaktik.html.

ECTS lietotāja rokasgrāmata. (2009). Retrieved from http://www.ec.europa.eu/education/lifelong-learning-policy/doc/ects/guide_Iv.pdf.

Eiropas kopīgās pamatnostādnes valodu apguvei: mācišanās, mācišana, vērtēšana. (2006). Rīga: Madonas poligrāfists, 220 S. Ipp. Retrieved from http://www.valoda. Iv/downloadDoc_419/mid_534.

Europäiche Charta für Mehrsprachigkeit. (2005). Retrieved from http://plurilinguisme. europe-avenir.com/images/Fondamentaux/charteplurilinguisme_dev2.13.pdf.

Franceschini, R. (2009). Inhalt/Contents. Retrieved from http://www.hueber.de/ .../ ForumSprache_01_2009_Artikel04-Franceschini_978-3-19-006100-6-2.pdf. 
Key Data on Teaching Languages at School in Europe (2008). Retrieved from: http:// eacea.ec.europa.eu/about/eurydice/documents/KDL2008_EN.pdf.

Königs, F. G. (2000). Fremdsprachen lehren und lernen. Tübingen: Gunter Narr Verlag , $259 \mathrm{~S}$.

Laicāne, M. (2007). Daudzkultūru izglīīiba projektā "NVO atbalsts patvēruma meklētājiem un bēgliem". Rīga: Patvērums. Drošā māja. Retrieved from: www. patverums-dm.Iv/svs/ uploads/files/Jun_daudzkulturu.rtf.

Meisel, J. (2006). Immersionsunterricht in Hamburger Grundschulen. In DIE ZEIT, 10/ 02. März, S. 37.

Meißner, F. J. (2004). EuroComprehension und Mehrsprachigkeitsdidaktik, 21 S. Retrieved from http://www.eurocomresearch.net/lit/meissner.pdf.

Ministru kabineta noteikumu projekta "Grozījumi Ministru kabineta 2008 gada 2 septembra noteikumos Nr. 715. (2012). Retrieved from http://www.likumi.lv/doc. php?id=242361.

Mitrofanova, L. (2012). 2011./2012. M. G. Valsts pārbaudes darbu vācu valodā rezultātu apkopojums. VISC Powerpoint.

Mūrnieks, A. (2011). Sabiedrības kultūras ietekme uz izglītības mērku veidošanu izglītības vadībā Latvijā. Rīga: $88 \mathrm{~S}$. Retrieved from http://www.lu.Iv/fileadmin/user_upload/ lu_portal/ zinas/ Andrejs_Murnieks_Promocijas_darba_kopsavilkums.pdf.

Pamatrādītāji par valodu mācīšanu Eiropas skolās. (2012). Retrieved from http://eacea.ec.europa.eu/education/eurydice/documents/key_data_series/143LV_HI.pdf.

Sabiedrības integrācijas politikas pamatnostādnes 2010-2019 gadam (2005). Retrieved from http://www.mk.gov.lv/doc/2005/BMPamn_210409_integr.633.

The Common European Framework of Reference for Languages, Learning, Teaching, Assessmet (CEFR) (2001). Council of Europe. Retrieved from: http://www.coe. int/t/dg4/linguistic/Source/Framework_en.pdf.

Valodas situācija Latvijā: 2004-2010. (2010). Retrieved from http://www.valoda.Iv/ downloadDoc_650/mid_510.

Valtenbergs, V. (2006). Attīstības izglītības kompetences Latvijā. Kā paaugstināsim savu jūtīgumu. Latvija un attīstības sadarbība. Zinātniski pētnieciskie raksti, Zinātne, 4 (10), (281-312), (359). Retrieved from http://www.mfa.gov.lv/data/ file/AttistibasSadarbiba/attistibas-sadarbiba.pdf.

Weißbuch zur allgemeinen und beruflichen Bildung. Lehren und Lernen. Auf dem Weg zur kognitiven Gesellschaft. (1995). 86 S. Retrieved from http://europa.eu/ documents/comm/ white_papers/pdf/com95_590_de.pdf.

\section{Rūta KEMERE}

Liepājas 5.vidusskola

\section{Alīda SAMUSEVIČA}

Liepaja University

\section{FOREIGN LANGUAGE LEARNING PROMOTING MULTILINGUALISM IN LATVIA}

Summary. The publication is devoted to the pupils' foreign language acquisition process development in the work of Latvian specialized language schools. The work of language specialization schools is an important resource of multilingualism promotion and development, 
which in the globalization conditions, when the geographic mobility increases, is able to improve the social quality of the society, promoting the transition from a homogeneous culture and value system to the culture pluralism and heterogeneity. The work of language specialization schools is structured by a pedagogical system which is flexible and open to education reforms when the paradigm of language acquisition changes.

The goal of the publication is to describe how to promote the quality of foreign language acquisition, on the grounds of the pedagogic work optimization in the German language diploma schools (DSD), in the context of multilingualism promotion.

In the publication the authors analyse the basic regulations of the language education policy in Latvia in order to stabilize multilingualism positions, describe the organizational and pedagogical aspects of the German language diploma schools' work in multilingualism development, emphasising the topicality of didactic and pedagogic diversity. The description of the pedagogic optimization and analysis in the DSD schools about the German language acquisition promotion are very important in order to be aware of the innovative pedagogical approach.

In the article the empiric research results about the pupils' motivation and quality of the language acquisition are analyzed, the young people's attitude and opinions in the context of multicultural society formation are described. Some conclusions are offered about multilingualism formation preconditions, making the strengthening of multilingualism positions topical in Latvia with the support of language specializations schools' (DSD schools) growth, accepting these schools as the democratic and didactic initiatives of multilingual centres. It is important to guarantee the pupils an opportunity to acquire several foreign languages simultaneously at school, allow the pupils to choose freely their first foreign language when starting the foreign language acquisition in the first form, as well as improve the assessment system of pupils' knowledge and skills in language learning, thus promoting the motivation of language acquisition.

The results of the empiric research prove clearly that the motivation of the pupils from the specialized language schools, their openness towards several foreign languages, their purposeful and qualitative acquisition already at school and its creative usage in their further life are the most significant language acquisition preconditions, which are topical for the youth and which will mostly determine the quality of multilingualism in Latvian society. It is important to achieve a sufficient assessment of the foreign language education role, which results from the democratic and human education paradigm Latvian focused on every personality's uniqueness, as well as the language wholeness and uniqueness.

Keywords: pupils, foreign language acquisition, language specialization schools, promotion of multilingualism.

\section{Rūta KEMERE,}

Liepojos 5-oji vidurinè mokykla

\section{Alīda SAMUSEVIČA}

Liepojos universitetas

\section{UŽSIENIO KALBU MOKYMASIS SKATINANT DAUGIAKALBYSTĘ LATVIJOJE}

Santrauka. Straipsnyje nagrinejjamos mokiniu užsienio kalbos issisavinimo apraiškos specializuotose kalbu mokyklose Latvijoje. Specializuotu kalbu mokyklu darbas - svarbus daugiakalbystės skatinimo ir plètojimo veiksnys, galintis globaliame pasaulyje, kuriame didejja geografinis mobilumas, pagerinti socialinès koreliacijos kokybę bendruomeneje, skatindamas peréjima nuo vienalytès kultūros ir vertybiu sistemos prie kultūrinio daugialypumo ir daugiakalbystès. 
Specializuotu kalbu mokyklu darbą struktūrizuoja pedagoginè sistema, kuri yra lanksti ir atvira ugdymo reformoms, kintant kalbu mokymosi paradigmai. Straipsnio tikslas - aprašyti, kaip skatinti užsienio kalbos issisavinimo kokybę daugiakalbystės kontekste, optimizavus pedagogini darbą vokiečiu kalbos diplominèse mokyklose (DSD). Straipsnyje autorès analizuoja pagrindinius kalbos mokymo politikos nuostatus Latvijoje siekiant stabilizuoti daugiakalbystès pozicija, apibūdinti organizacinius ir pedagoginius vokiečiu kalbos diplominiu mokyklu darbo aspektus plètojant daugiakalbystę, pabréžiant didaktinès ir pedagoginès ¡vairovès svarba. Norint išsiaiškinti inovatyvius pedagoginius metodus, labai svaru aprašyti pedagogini optimizavimą ir analizę, susijusią su vokiečiu kalbos mokymosi skatinimu DSD mokyklose.

Straipsnyje analizuojami empirinio tyrimo apie mokiniu motyvacija ir kalbos isisavinimo kokybę rezultatai, taip pat aprašomas mokiniu požiūris ir nuomonès dèl daugiakultūrès visuomenės kūrimo. Pateikiamos išvados apie išankstines daugiakultūriškumo formavimo salygas siekiant sustiprinti jo pozicija Latvijoje ir paremti specializuotu kalbu mokyklu (DSD) daugejjima, pripažistant šias mokyklas demokratinès ir didaktinès iniciatyvos daugiakultūriais centrais. Svarbu užtikrinti mokiniams galimybę tuo pačiu metu mokykloje mokytis keliu užsienio kalbu, leisti laisvai pasirinkti pirmaja užsienio kalbą ir pradèti jos mokytis pirmoje klaseje, taip pat pagerinti žiniu ir kalbos igūdžiu vertinimo sistemą keliant motyvacija mokytis kalbu.

Tyrimo rezultatai irodo, kad specializuotu kalbu mokyklu mokiniu motyvacija, atvirumas kelioms užsienio kalboms, tikslingas ir kokybiškas kalbos mokymasis dar mokykloje ir kūrybiškas jos vartojimas ateityje yra svarbiausios išankstinès kalbos mokymosi salygos, aktualios jaunimui ir labiausiai nulemsiančios Latvijos visuomenès daugiakalbystès kokybę. Sukurti tinkamą požiūri i užsienio kalbos ugdymą - svarbi užduotis, kuri yra demokratinio ir humanistinio ugdymo paradigmos, orientuotos i kiekvienos asmenybès ir kalbos unikaluma, išdava.

Pagrindinès sąvokos: mokiniai, specializuotos kalbu mokyklos, daugiakalbystès skatinimas. 